\title{
WHAT IS THE BEST WAY TO DEAL WITH MALIGNANT BRAIN TUMORS
}

\author{
Fraser Sacus *
}

Up to the present time, the only method we have of removing hrain tumors is by surgery. We all hope the time may come when some other method may be discovered but until such time arrives, we must continue to resort to surgery. Every one is agreed that in the case of benign tumors this is the proper procedure but a difference of opinion has developed recently in regard to malignant tumors. As a result two groups have developed.

One takes the attitude that since these tumors are malignant and there is no positively recorded cure, it is not worthwhile to try to operate on these patients; this a defeatist attitude with which I never have had any sympathy. We all recall conditions which when first treated gave very unsatisfactory results; had men stopped treating them we would have made no progress and surgery would have remained at a standstill. Then too we must consider can we be positive we are dealing with a malignant tumor. There are neuro-roentgenologists who claim to be able to determine the type of tumor by an angiogram. Though this may be possible at times, the only way to determine this positively is to get a histological examination of the tumor, and that can be obtained only by some form of operation. To subject a patient to operation in order to get a biopsy and then to close the wound because we find a malignant tumor is a very unsafe procedure; such patients do very badly and the associated oedema leads as a rule to a rapid fatality. A needle biopsy is not attended by as much risk but the result is equally unsatisfactory.

The other group feels that even if one cannot accomplish a permanent cure, it is well worthwhile to give a patient temporary relief. These surgeons feel that is well worthwhile to relieve patients of intolerable headache, to save their eyesight and to rehabilitate them for a time at least, sometimes as much as a year, so that they can carry on their former work; the men who adopt this method remove the tumor as completely as

Palestra pronunciada durante a visita que o Prof. Ernest Sachs fêz ao Serviço de Neurologia da Fac. Med. da Univ. de São Paulo, em fevereiro 1952.

* Research Associate in Physiology, Yale Iniversity, New Haven; Formerly Professor of clinical neurological surgery Washington University School of Merlicine, St. Iouis. 
they can and give the patient an internal decompression in order to relieve the pressure symptoms as long as possible. By following this policy there is the opportunity of being more radical at operation. Ultimately we may be able to improve our technique to such an extent that permanent cures may be affected. Is this a possibility with the radical method?

To determine that we must review the progress in the last 30 vears. Our greatest problem used to be the localization of a tumor. The only method we had up to that time was a careful neurological history and physical examination. In the very best hands 55 per cent of tumors at most were localized, but with the discovery of ventriculography this picture changed completely and after the method came into geveral use fully 97 per cent of all tumors were located. With the free use of angiography the percentage of correct diagnosis has been still further increased. The day has passed when after subjecting patients to craniotomy the surgeon had to admit repeatedly he had been unable to expose the tumor.

With this improvement in diagnosis, there also has been a corresponding improvement in technique. This has been accomplished by the use of electrosurgery, suction, better hemostasis with fibrin foam or gelfoam and the free use of blood transfusion. Then came the question of how to deal with ill-defined and non-encapsulated tumors. At this point a most important further additions was made to our aryemantarium. In 1926 Cushing and Bailey, using Hartega's new staining methods, were able to differentiate the various types of gliomas and to determine which were malignant and which were not. The degree of malignancy varies in the different types; oligodendrogliomas, medulloblastomas and ependymomas all have a tendency to recur though they may grow very slowly and, by repeated operations, the patients may survive for many years. The life of a patient with a medulloblastoma may be prolonged with the use of deep X-ray therapy since that type of tumor is very radiosensitive, but the most malignant type is the glioblastoma and this is the tumor that $-t i l$ ! gives us the greatcst concern.

The next step was to review the histories of these various tumor types and determine whether clinically we could distinguish between them. It quickly became apparent that a glioblastoma grew more rapidly and the symptoms developed more quickly than with other tumors but excep: for this there was no positive way of determining the lype of patho'ogy we were dealing with. Glioblastomas occur in the vast majority of cases in the cerebrum; thus in a study of 240 cases of glioblastomas, $23: 3$ nere found in the cerebrum, nevertheless it still was necessary to operate to establish the pathology definitely. No treatment should be undertaken until we know the pathology. To give deep X-ray therapy without knowing the pathology of the tumor is very unwise and I think is a great mistake.

Today having the advantage of these diagnostic and technical aids we are far better able to deal with a glioblastoma than we used to be. 
Still we must decide whether it is worthwhile to give a patient relief that can be only temporary. This brings up the ethical aspect. What is the prime function of a physician? Should he attempt to relieve a patient only when he feels he can effect a permanent cure or should he content himself with temporary relief if he is unable to accomplish a cure. To my mind there is no argument here. For both medical and ethical reasons we must keep on trying to help for only in this way can we continue to advance. Of course we would always like to effect a cure but if this is not possible the patient is entitled to relief by a palliative procedure. This has been the policy that has been followed with malignancy elsewhere in the body: carcinoma of the stomach today has a far better prognosis than it had 20 years ago and the same may be said about other malignant processes.

A favorable aspect of brain tumors is that they do not metastasize. Our greatest difficulty has been to find the limits of a malignant brain tumor. Within the past two years however a new technique has been developed which gives promise of overcoming this obstacle. With the injection of radioactive isotopes the limits of a tumor can be much more accurately determined. Thus a more radical removable becomes possible and the prospect of a permanent cure seems more likely.

In a recent review of my cases I find that the longest survival occured in those cases in which radon seeds were implanted after a radical removable had been carried out. The use of radioactive isotopes and implanting radon seeds seem to me to be convincing proof, if more were needed, that we must try to remove malignant brain tumors, for silting by and doing nothing has never yet led to progress.

$$
\text { Yale University - New Haz'en, U.S.A. }
$$

